\title{
Warm thick target solar $\gamma$-ray source revisited
}

\author{
A. L. MacKinnon ${ }^{1}$ and M. P. Toner ${ }^{2}$ \\ 1 Department of Adult and Continuing Education, University of Glasgow, Glasgow G3 1LP, UK \\ 2 Department of Physics and Astronomy, University of Glasgow, Glasgow G12 8QQ, UK \\ e-mail: mark@astro.gla.ac.uk
}

Received 1 July 2002 / Accepted 18 June 2003

\begin{abstract}
The $1.63 \mathrm{MeV} \gamma$-ray line of ${ }^{20} \mathrm{Ne}$ is sensitive to protons of lower energies than most other nuclear de-excitation lines. Its unexpected strength has been taken as evidence for a solar flare fast ion distribution that remains steep at low energies, and thus has a large total energy content. It has also been suggested that its strength might instead reflect the enhancement of ion lifetimes that occurs when ambient temperatures exceed $10^{7} \mathrm{~K}$. Here we revisit this idea (a) recognising that ions may be effectively trapped in high temperature regions and (b) taking account of the contribution to the line of all ions above threshold. The strength of the $1.63 \mathrm{MeV}$ line relative to other de-excitation lines has been used to estimate the steepness (e.g. energy power-law index) of the ion distribution. We show that these estimates must be significantly revised if primary ions are contained in a region with temperature in the few $10^{7} \mathrm{~K}$ range, lower than found elsewhere. Such a region would almost certainly be coronal, so we also briefly review other arguments for and against coronal $\gamma$-ray sources.
\end{abstract}

Key words. Sun: flares - Sun: X-rays, $\gamma$-rays - plasmas - acceleration of particles

\section{Introduction}

Accelerated, nonthermal particles are a primary product of the energy release process in solar flares. We have known for decades that the energy content of electrons above about 20-25 keV appears to constitute several tenths of the total energy manifested in the flare (e.g. Hoyng et al. 1976; Lin \& Hudson 1976), but the situation in respect of ions is less wellestablished. Early, SMM era estimates of the energy content of ions above $1-10 \mathrm{MeV} /$ nucleon (e.g. Murphy \& Ramaty 1984; Ramaty 1986) indicated values modest compared to those of electrons. The total energy content of ion distributions falling off steeply with energy is dominated by the behaviour at the lowest energies. These conclusions were mostly reached in the context of the Ramaty-Lee model for particle acceleration (Ramaty 1986), which predicts a distribution that flattens at low energies in such a way as to have a finite total energy without any need for an arbitrary, low-energy cutoff. Various lines of argument suggested that this form of distribution was preferred by observations (e.g. Murphy \& Ramaty 1984). The situation has changed more recently, however.

In particular, Share \& Murphy (1995) found that the observed flux of photons in the $1.63 \mathrm{MeV}$ line due to deexcitations of ${ }^{20} \mathrm{Ne}$ was stronger than expected on the basis of previous estimates of ion energy distribution, even allowing for an enhancement of the $\mathrm{Ne}$ abundance over its photospheric value. The $1.63 \mathrm{MeV}$ state of ${ }^{20} \mathrm{Ne}$ is excited by protons of

Send offprint requests to: A. L. MacKinnon,

e-mail: a.mackinnon@educ.gla.ac.uk energies of $2 \mathrm{MeV}$ and above, a lower threshold energy than that appropriate to most other de-excitation lines. Thus the unexpectedly strong $1.63 \mathrm{MeV}$ line might reflect a fast proton distribution declining with energy more steeply than expected. In particular it appeared possible that the distribution falls off more steeply than the Bessel function $K_{2}$ in momentum predicted by the Ramaty-Lee model, and thus that total fast ion energy content is much greater than the SMM era estimates (Ramaty et al. 1996). For example, Murphy et al. (1997) deduced a total energy of $1.7 \times 10^{32}$ ergs in protons above $1 \mathrm{MeV}$, and $10^{33} \mathrm{ergs}$ in fast ions of all species (assuming these are all described by the same form of energy distribution).

All of these discussions also assume thick target production of $\gamma$-rays, i.e. that the accelerated ions stop completely in the source. Then the energy loss rate of the fast ions also enters the discussion, because it determines how long they stay above any particular energy and thus the number of photons they can produce while slowing down. Ions may interact resonantly with MHD waves but this results primarily in pitchangle scattering, at least to first order (e.g. Miller \& Ramaty 1987), rather than any change in energy. Thus the necessary energy loss rates are given by test particle treatments of binary interactions (e.g. Spitzer 1956; Trubnikov 1965). Emslie et al. (1997) pointed out that all the existing treatments assumed "cold target" energy loss rates, assuming test particle speeds are $\gg$ target particle speeds. For temperatures $>10^{7} \mathrm{~K}$ and proton energies $\sim$ a few $\mathrm{MeV}$, however, this may no longer be the case. In this "warm target" regime fast ions lose less energy in each binary collision, their effective lifetimes in the source 
are longer, and their photon yields potentially enhanced. Thus the stronger than expected $1.63 \mathrm{MeV}$ line might reflect a reduced, warm target energy loss rate near threshold, rather than a greater number of fast protons.

Emslie et al. (1997) eventually concluded that warm target effects could not account for the ${ }^{20} \mathrm{Ne}$ line enhancement, however, because the diminution in energy loss rate compared to cold target conditions simply allowed the protons to leave the hot coronal region more easily, passing rapidly to chromospheric regions where they would produce their cold target photon yields, as previously discussed. However this conclusion is reached neglecting any other factors that could contain protons in the corona, e.g. pitch-angle scattering by Alfvén waves (Tamres et al. 1989), and a discussion of a hypothetical, fully contained source was carried out only in a semiquantitative way.

Only a radical departure from conventional wisdom would locate a $\gamma$-ray warm target anywhere other than the corona. In the absence of such a departure, we need to note that there are flares in which a significant fraction of observed $\gamma$-ray line production does indeed appear to take place in the corona (Barat et al. 1994; Vestrand \& Forrest 1993), but also strong arguments against a nearly isotropic, coronally trapped population in other flares (e.g. temporal behaviour apparently requiring rapid precipitation, Hua et al. 1989; redshifted $\gamma$-ray lines indicative of significant anisotropy, Share et al. 2002). The situation appears unclear at present, but we may say that a coronal, possibly warm target origin for $\gamma$-ray lines appears possible in at least some events, and thus that warm target effects may be important in interpreting $\gamma$-ray line fluxes from at least some events (see also Sect. 4).

Here we revisit the possible effect of warm target energy losses on the $1.63 \mathrm{MeV}{ }^{20} \mathrm{Ne}$ line. Section 2 briefly reviews some of the work of others which demonstrates that fast ions may well contain themselves in the corona, in particular by generating Alfvén waves. Section 3 evaluates the effect of warm target energy losses on the yield of the $1.63 \mathrm{MeV}$ line in a hypothetical, fully contained source, emphasising the importance of considering all relevant proton energies. Such a hypothetical source may not correspond with reality but it does allow us to determine the maximum influence warm target effects could ever have on estimates of ion energy distribution and total energy content. Section 4 briefly states our conclusions and discusses one or two relevant issues, in particular noting the possibility of a dependence of relative $\mathrm{Ne}$ line strength on flare size. A brief appendix reviews theoretical and experimental results on ion energy loss rates in neutral and ionised targets.

\section{Proton containment in the corona}

In what follows we assume the usual picture of a low-lying, flaring magnetic loop, with a comparatively tenuous (typically $10^{10}-10^{11} \mathrm{~cm}^{-3}$ ) coronal region where particle acceleration takes place. Both ends of coronal field lines are anchored in a denser, cooler chromospheric region where $\gamma$-ray producing ions will stop and produce most of their radiation, if otherwise unimpeded. Warm target conditions, if they apply at all, will be relevant in the corona, so we wish here to note any factors which will increase the coronal residence time of fast protons.

First, and most trivially, protons which are given large pitch angles $\theta$ at the time of their acceleration will spend a time $1 / \mu$ longer in the corona, where $\mu=\cos \theta$ (ignoring any effects of magnetic field inhomogeneity). Barring the a priori unlikely circumstance of a concentration at large pitch angles, however, this will increase coronal residence times by a factor only of order unity.

Pressure balance arguments at least suggest magnetic field strength will increase from corona to chromosphere. The resulting trapping, in a coronal magnetic bottle, has often been discussed. Denote by $B_{0}$ the magnetic field strength at the apex of the loop, and by $B_{1}$ the field strength at a footpoint, i.e. at the depth in the atmosphere beyond which collisions will prevent all but a negligible fraction of incident protons from mirroring and returning. In the absence of pitch-angle scattering, any protons with pitch angles greater than $\theta_{0}=\sin ^{-1}\left(B_{0} / B_{1}\right)^{1 / 2}$ are trapped in the corona. For an isotropic distribution, a fraction $\mu_{0}=\cos \theta_{0}$ of all accelerated protons are trapped in this way. Only a modest field strength variation is needed to trap a significant fraction of all protons: $\mu_{0}=0.5$, for instance, implies just $B_{0} / B_{1}=0.75$.

These considerations apply to ions produced in a coronal loop by some unspecified mechanism. More effective trapping may occur naturally in more involved geometries (e.g. Lau et al. 1993). In particular, ions accelerated in reconnection near a coronal null may be much more effectively trapped because they begin to move adiabatically at very low field strengths (Fletcher \& Martens 1998).

While binary collisions are ineffective at scattering fast ions (Emslie 1978), other mechanisms for more effective pitchangle scattering may well operate. In particular, protons with speeds greater than the Alfvén speed, $v_{\mathrm{A}}$, may interact resonantly with Alfvén waves (e.g. Kennel \& Petschek 1966). A proton moving at the Alfvén speed has energy $E$ (in $\mathrm{keV}$ ) given by

$E=25 B_{2}^{2} / n_{10}$

where $n_{10}$ and $B_{2}$ measure ambient density and field strength in units of $10^{10} \mathrm{~cm}^{-3}$ and $100 \mathrm{~s}$ of $\mathrm{G}$, respectively. Thus all protons of interest for $\gamma$-ray production may potentially interact with Alfvén waves, as has been discussed in detail elsewhere (e.g. Miller \& Ramaty 1987). The relative magnitudes of scattering $\tau_{\mathrm{s}}$ and loop transit $\tau_{1}$ times determine the regime in which scattering takes place, and thus its effects on coronal containment (Kennel \& Petschek 1966; Bespalov et al. 1987): weak $\left(\tau_{\mathrm{s}} \gg \tau_{1}\right)$, in which scattering reduces residency times by allowing particles to trickle into the loss cone; moderate $\left(\tau_{\mathrm{s}} \simeq \tau_{1}\right)$, in which the distribution remains close to isotropic and a fraction $\left(1-\mu_{0}\right)$ of particles can precipitate; strong $\left(\tau_{\mathrm{s}} \ll \tau_{1}\right)$, in which scattering actually increases coronal residency times, even for particles inside the loss cone, by impeding the free flow of particles out of the loop.

Hua et al. (1989) carried out studies of proton turbulent transport in converging loops, treating scattering with a Monte Carlo method. Restricting their simulations to cases in which the isotropisation length was at least twice the loop 
length, they confirmed the existence of the weak and moderate regimes, finding a saturation of the precipitated flux as scattering time reduces to the point that the distribution is close to isotropic. Situations with still shorter mean free paths were (Monte Carlo) simulated by Kocharov et al. (1999), confirming the existence of the strong regime with its enhanced containment.

The strong regime might arise in flares in (at least) one of two ways. First, Alfvén turbulence might itself be the means by which ions are accelerated to $\gamma$-ray producing energies. In this case, the turbulent energy density needed to accelerate protons fast enough implies scattering in the strong regime, and indeed effective containment in the acceleration region (Smith 1990). Second, and most importantly, very modest anisotropies of the large numbers of protons needed to produce observed $\gamma$-ray fluxes are adequate to result in unstably growing waves (Tamres et al. 1989; Bespalov et al. 1987). Even if produced isotropically, such anisotropies will inevitably occur as protons travel away, with different parallel velocities, from a localised source region. The number of protons involved in the June 7 , 1980 flare, for instance, evidently implies turbulent propagation of protons in the strong scattering regime (Smith \& Brecht 1991), with coronal residency times many times greater than loop transit times. Kocharov et al. (2000) have also drawn attention to the possible role of self-generated Alfvén turbulence in containing ions in warm target regions.

It seems clear that a variety of factors point to typical coronal residency times substantially greater than the loop transit times used in Emslie et al. (1997), and thus to the possibility, at least that large numbers of $\gamma$-ray producing protons slow down mostly in a warm target region. In the next section we explore the consequences of this possibility quantitatively, in the limit that the containment mechanism has no significant influence on ions' evolution in energy. Before doing this we also note that other coronal containment mechanisms have been proposed, in the presence of which a more elaborate re-evaluation of radiative yields would be necessary - e.g. Spicer and Emslie (1988).

\section{Warm target effects in $\gamma$-ray line strengths}

\section{1. $\gamma$-ray line flux in a target of arbitrary temperature}

In this section we assume for illustration that all protons slow down in a fully ionised plasma characterised by a single, ambient temperature $T$. We will consider the effect of $T$ on the yields of various $\gamma$-ray lines. Emslie et al. (1997) found that implausibly high values of $T$, in excess of $10^{8} \mathrm{~K}$, would be necessary to yield a significant enhancement of ${ }^{20} \mathrm{Ne}$ line flux, even with perfect coronal trapping. First we look briefly at how this conclusion was reached and argue that it deserves to be revisited.

The energy loss rate of a proton in a fully ionised hydrogen plasma of temperature $T(\mathrm{~K})$ is given by (e.g. Trubnikov 1965 ; Tamres et al. 1986):

$$
\frac{\mathrm{d} E}{\mathrm{~d} t}=-f(E) n \Theta(E)
$$

where

$f(E)=\frac{2 \pi e^{4} Z^{2} \Lambda(2 m)^{1 / 2}}{m_{\mathrm{e}} E^{1 / 2}}$

where $m$ and $Z$ are the mass (gm) and charge (multiples of the elementary charge $e$ ) of the incident fast ion, $m_{\mathrm{e}}$ is the electron mass, $\Lambda$ is the Coulomb logarithm (dependent on energy and species, and given for fast particles e.g. by Emslie 1978; see also the Appendix $), n\left(\mathrm{~cm}^{-3}\right)$ is the ambient particle density and $\Theta(E, T)$ is the ratio of the true energy loss rate to that experienced in a cold target (i.e. a source region in which $T \rightarrow 0$ ). $\Theta \rightarrow 1^{-}$as $E / k T \rightarrow \infty$ and the details of its evaluation can be found in Tamres et al. (1986).

We need not carry out any drastic reconsideration of the ions' energy evolution in the case that we appeal to interaction with Alfvén waves for coronal containment. For typical loop lengths $\sim 10^{9} \mathrm{~cm}$ and proton energies of a few $\mathrm{MeV}$, values of $\tau_{\mathrm{s}} \sim 1 \mathrm{~s}$ are in the strong scattering regime. Changes of proton energy due to interaction with Alfvén waves take place on a time $\sim\left(\frac{c}{v_{\mathrm{A}}}\right)^{2}$ longer (e.g. Kulsrud \& Pearce 1969), easily in the $10^{3}-10^{4} \mathrm{~s}$ regime for typical field strengths and densities and much longer than collisional slowing down times.

If we inject a population of protons with energy distribution $F(E)$ (i.e. the number of protons with energies between $E$ and $E+\mathrm{d} E$ is $F(E) \mathrm{d} E$ ), then the standard thick target formalism for $\gamma$-ray line production (e.g. Ramaty 1986) tells us that the total number of photons $\Phi_{k}$ produced in line $k$ is

$\Phi_{k}=a_{k} \int_{E_{k}}^{\infty} \frac{v(E) \sigma_{k}(E)}{f(E)} \Psi(E) \mathrm{d} E$

where $E_{k}$ and $\sigma_{k}$ are the threshold energy and cross-section (as a function of incident proton energy) for production of line $k$, $a_{k}$ is the abundance relative to hydrogen of the target nuclei and $\Psi(E)$ is the total number of protons injected above energy $E$, i.e.

$\Psi(E)=\int_{E}^{\infty} F(E) \mathrm{d} E$.

The dominant, direct excitation cross-section for the particular line of interest, the $1.63 \mathrm{MeV}$ line, has its threshold at $\sim 2 \mathrm{MeV}$ and its maximum value at $6.4 \mathrm{MeV}$. Cross-sections generally climb steeply from threshold so one might expect the total (proton-excited) yield of $1.63 \mathrm{MeV}$ photons to be dominated by protons about 6-7 MeV. Inspection of the integrand of Eq. (4) for the particular case of this line is instructive, however. Even for hard injected spectra, the contribution from protons immediately above threshold is significant compared to that from protons of 6-7 MeV. Thus it is important to include the full proton energy range all the way from threshold.

Emslie et al. (1997) carried out detailed calculations only for the case where no non-collisional factors lengthen coronal lifetimes. They did consider a source region characterised by a single temperature, but discussed such a source only semiquantitatively, basing rough arguments on the enhancement of proton lifetime expected for protons of energies around the maximum of the $1.63 \mathrm{MeV}$ line cross-section. Since even protons immediately above the threshold of the cross-section can 
make a significant contribution, particularly for power-law ion distributions which fall off steeply with energy, a more detailed discussion is needed.

Now we ask the following question: for protons of a given energy $E$, what does the source temperature $T$ have to be to reduce the energy loss rate by one order of magnitude, i.e. to give $\Theta=0.1$ ? The factor of 0.1 is quite arbitrary but serves to make our point. In the "warm target" regime

$$
\frac{m_{\mathrm{p}}}{m_{\mathrm{e}}} \gg \frac{E}{k T} \gg 1
$$

a simple, analytical approximation for $\Theta$ exists (Tamres et al. 1986), and we can easily show

$k T=2.1 E$

where $k T$ has been expressed in $\mathrm{keV}$ and $E$ in $\mathrm{MeV}$. As found by Emslie et al. (1997), such a reduction of proton energy loss rate at $7 \mathrm{MeV}$ needs very high temperatures $\left(\sim 2 \times 10^{8} \mathrm{~K}\right)$. Nearer the threshold of the cross-section however, at $2-3 \mathrm{MeV}$, a more acceptable temperature of $7 \times 10^{7} \mathrm{~K}$ will do. Several observations exist suggesting flare temperatures in the range $10^{7}$ to $10^{8}$ K (e.g., Tsuneta et al. 1997; Warren and Reeves 2001).

Thus a complete assessment of the influence of warm target effects on $\gamma$-ray line yields must consider the contribution of all proton energies above threshold, not just those in the vicinity of the cross-section maximum. Also "gradual phase" flare conditions can extend the lifetime of trapped protons near threshold by up to an order of magnitude.

\subsection{Role of $\alpha$ particles}

As pointed out by Share and Murphy (1995), too many $\alpha$ particles in the accelerated population complicates the interpretation of the $1.63 \mathrm{MeV}$ line. The cross-section data presented in Ramaty et al. (1979; see also Dyer et al. 1981, 1985; Seamster et al. 1984) clearly show that this line's threshold when excited by $\alpha$ 's, rather than protons, is not significantly different from that of other de-excitation lines. So, if $\alpha$ 's are overabundant in the fast ion distribution - as appears to be the case in at least some flares (Murphy et al. 1991; Share \& Murphy 1998) - assuming an injected fast ion distribution extending steeply to low energies helps less with understanding the observed 1.63 MeV line flux. For example, Murphy et al. (1997) used the ratio $\Phi_{1.63} / \Phi_{6.13}$ to deduce $\delta$ values of 4.0 and 4.4 assuming a relative abundance ratio $\alpha / p$ of 0.1 and 0.5 respectively. Clearly the assumption of identical energy distributions for protons and $\alpha$ 's, combined with the deleterious effect of $\alpha$ 's on the $1.63 \mathrm{MeV}$ flux relative to other lines, combine to push the deduced ion distribution to steeper forms. Larger and larger values for the total ion energy content follow immediately.

In general, thresholds for excitation by alphas are of the same order as thresholds for protons when expressed in $\mathrm{MeV} /$ nucleon. It follows immediately that warm target effects will be of a similar order of magnitude and thus we include them explicitly in these calculations. As summarised above, inclusion of alphas in cold target calculations softens the deduced ion spectrum, increasing the total ion energy. The precise magnitude of this effect in the warm target case will depend on the detailed forms of the cross-sections and can only be determined by exact calculation.

\subsection{Results}

In Fig. 1 we show the ratio $\Phi_{1.63} / \Phi_{6.13}$ as a function of $T$, calculated as described in the previous section. We assume that $F(E)$ has power-law dependence on $E, F(E) \sim E^{-\delta}$, up to $E=E_{\max }$, and that it is zero for $E>E_{\max }$. We adopted $E_{\max }=1 \mathrm{GeV}$ throughout; as long as $\delta>2$ and $E_{\max } \gg 100 \mathrm{MeV}$ its value has no important influence on the results. We have assumed $\alpha / p=0.1$, a range of values of $\delta$ and the same target isotopic abundances as Ramaty et al. (1996). In particular the assumed ${ }^{20} \mathrm{Ne} /{ }^{16} \mathrm{O}$ abundance ratio is 0.14 . At the larger $\delta$ 's the results of Figs. 1, 2 and 3 scale roughly linearly with ${ }^{20} \mathrm{Ne} /{ }^{16} \mathrm{O}$ abundance ratio. Spallation reactions on other species contribute up to $30 \%$ of the $1.63 \mathrm{MeV}$ flux at the hardest $\delta$, however, complicating the effects of abundance variations.

In common with all previous work, we assume that proton and $\alpha$ distributions are characterised by the same value of $\delta$, and that the abundance ratio $\alpha / p$ refers to the relative numbers of particles above the same energy per nucleon. These assumptions will be submitted to scrutiny elsewhere. Cross sections come from Dyer et al. $(1981,1985)$ and Seamster et al. (1984), as far as we know the most recent, published measurements (see also Kozlovsky et al. 2002). We include contributions to the total line fluence from both direct excitation of e.g. ${ }^{20} \mathrm{Ne}$ nuclei for the $1.63 \mathrm{MeV}$ line, as well as the various spallation type channels which can contribute to the lines (listed in Kozlovsky et al. 2002). Ramaty et al. (1996) previously calculated $\Phi_{1.63} / \Phi_{6.13}$ assuming ions slow down in a cold, neutral target. We checked that our numerical evaluation of Eq. (4) gives the same results when the same energy-loss rate $f(E)$ (empirically derived in Barkas \& Berger 1964) is used. These are similar to our ionised medium results in the case $(T \rightarrow 0)$ but not identical because of differences in the energy-dependence of $\Lambda$ in the neutral and ionised cases; see the Appendix. They also give us reference results for discussion of the warm target case.

We see that temperatures in excess of $10^{7} \mathrm{~K}$ lead to enhanced values of $\Phi_{1.63} / \Phi_{6.13}$. Warm target effects have least influence on $\Phi_{1.63} / \Phi_{6.13}$ for the hardest injected spectra.

Figure 2 shows similar results, now assuming $\alpha / p=0.5$. As anticipated, a greater relative $\alpha$ abundance in the fast ion distribution does make $\Phi_{1.63} / \Phi_{6.13}$ slightly less dependent on $T$, but a significant variation remains.

The ratio $\Phi_{1.63} / \Phi_{6.13}$ does not increase indefinitely with source temperature. In fact a maximum value occurs at a temperature weakly dependent on $\delta$ but generally around 4$5 \times 10^{8} \mathrm{~K}$. Figure 3 shows the maximum value of $\Phi_{1.63} / \Phi_{6.13}$ as a function of $\delta$, for a range of abundances of fast $\alpha$-particles relative to protons. The range of $\delta$ consistent with a given, observed $\Phi_{1.63} / \Phi_{6.13}$ is bracketed by the values obtained assuming a cold target (e.g. Ramaty et al. 1996; the extreme left-hand edges of Figs. 1 and 2), and the maximum possible warm target enhancement (Fig. 3). The $\alpha / p$ ratio may be established from, 


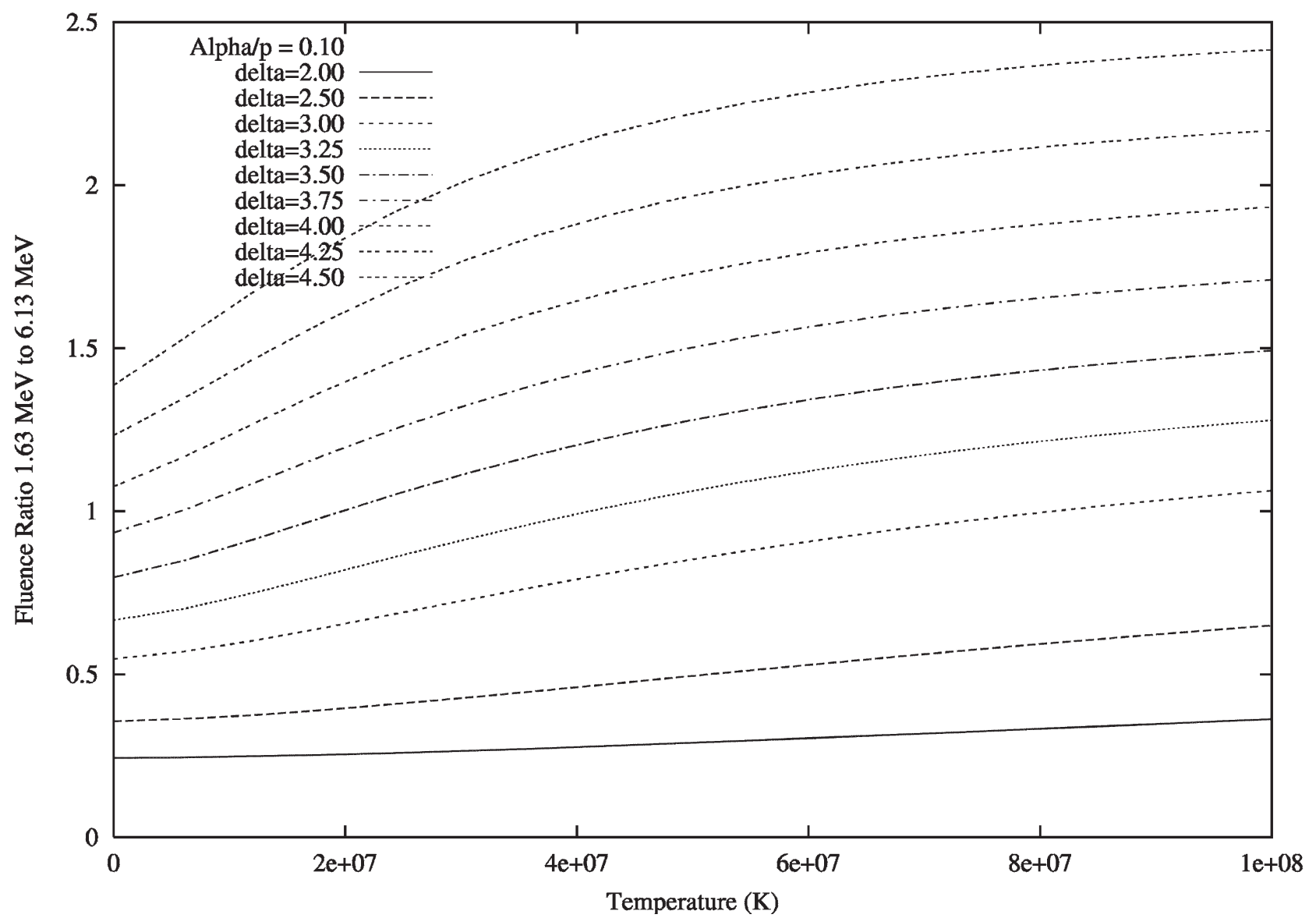

Fig. 1. Dependence of the ratio of line fluxes $\Phi_{1.63} / \Phi_{6.13}$ on $T$ for injected ion energy distributions characterised by various values of ion energy spectral index $\delta$. The fast $\alpha$-particle abundance relative to protons is $\alpha / p=0.1$.

say, analysis of $\alpha-\alpha$ lines such as the ${ }^{7} \mathrm{Be}$ and ${ }^{7} \mathrm{Li}$ lines in the $0.4-0.5 \mathrm{MeV}$ range (Murphy et al. 1990). With given $\alpha / p$, measured values lying above the curve of Fig. 3 demand relative abundances of ${ }^{20} \mathrm{Ne}$ and ${ }^{16} \mathrm{O}$ that differ significantly from the values assumed here.

\subsection{Examples of applications}

At first sight it might seem that such a contained source would allow us to interpret measurements of $\Phi_{1.63} / \Phi_{6.13}$ in terms of a flatter ion distribution than that found for a cold target, and thus one containing fewer ions and less energy in total. However, the Coulomb logarithm $\Lambda$ tends to be greater in an ionised target (Appendix). Specific examples are necessary to see whether these two effects conspire to imply increased or decreased ion numbers, compared to a cold, neutral target.

Share \& Murphy (1995) found values of $\Phi_{1.63}$ and $\Phi_{6.13}$ in 19 flares observed by SMM between 1981 and 1989 . Most values of the fluences in these lines lay in the range 10-30 photons. $\mathrm{cm}^{-2}$, with most associated values of $\Phi_{1.63} / \Phi_{6.13}$ in the range 1.0 to 1.4 . Taking a typical value of $\Phi_{1.63}=18$ photons.cm ${ }^{-2}$ and $\Phi_{1.63} / \Phi_{6.13}=1.4$, representing the high end of the observed range, we now give some illustrations of how a warm target source could influence deductions of flare ion energy distribution and total content.
First we discuss the reference case of a cold, neutral target. We illustrate the influence of fast $\alpha$ abundance by considering two cases, with fast $\alpha$ to proton abundances (denoted $\alpha / p$ ) of 0.1 and 0.5 (i.e. the number of $\alpha$ 's above $1 \mathrm{MeV} /$ nucleon is 0.1 or 0.5 times the number of protons above $1 \mathrm{MeV}$ ). The above combination of $\Phi_{1.63}$ and $\Phi_{6.13}$ are produced for $\delta \simeq 4.4$ $(\alpha / p=0.1)$ or $\delta \simeq 5.3(\alpha / p=0.5)$. Denote by $N_{\mathrm{p}}$ the number of protons above $1 \mathrm{MeV}$. Corresponding values of $N_{\mathrm{p}}$ are $7 \times 10^{36}$ and $9 \times 10^{36}$ for $\alpha / p=0.1$ and 0.5 , respectively, with numbers of alphas implied by $\alpha / p$. Note how assuming a greater abundance of fast $\alpha$ 's forces us to a steeper energy distribution, and thus to greater numbers of accelerated ions.

Now compare with the case of a fully ionised but cold target. Our illustrative line fluences are produced by $\delta \simeq 4.5$ and $N_{\mathrm{p}} \simeq 2.7 \times 10^{37}(\alpha / p=0.1) ;$ and $\delta \simeq 5.4$ and $N_{\mathrm{p}} \simeq 3.6 \times 10^{37}$ $(\alpha / p=0.5)$. The ion energy distributions are close to those found assuming a neutral target, but the different values of $\Lambda$ mean significantly greater numbers of ions are needed.

Next we consider the influence of a high temperature, contained source. With $\alpha / p=0.1$, a temperature of just $2 \times 10^{7} \mathrm{~K}$, quite in line with inferences from other data, lets us interpret the adopted fluences as resulting from $\delta \simeq 4$ and $N_{\mathrm{p}} \simeq 6.4 \times 10^{36} \mathrm{~K}$. No more ions are needed than in the neutral target case, but the ion energy distribution is harder. An even hotter source would imply a still harder distribution, with 


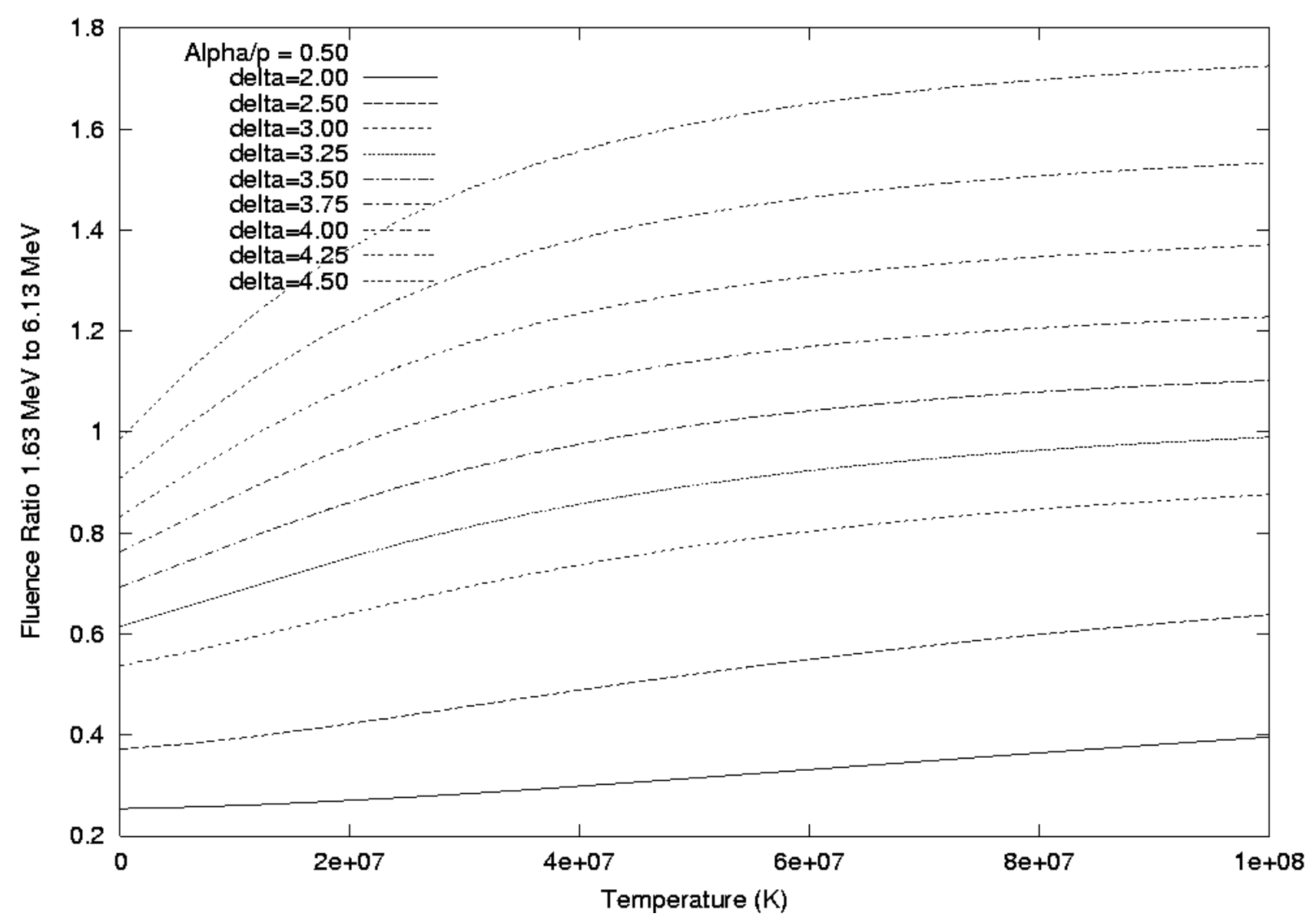

Fig. 2. As Fig. 1 except $\alpha$ 's are over-abundant: $\alpha / p=0.5$.

correspondingly reduced ion numbers and energy, e.g. for $T=$ $6 \times 10^{7} \mathrm{~K}$ and $\delta=3.5, N_{\mathrm{p}} \simeq 10^{36}$, almost an order of magnitude down on those found in the neutral target.

Observations of the $\mathrm{Li}$ and $\mathrm{Be}$ formation lines give independent information on flare $\alpha$ acceleration, often appearing stronger than expected and indicating an enhanced fast $\alpha$ abundance, e.g. $\alpha / p=0.5$ (Murphy et al. 1991; Share \& Murphy 1998). It is clear from Fig. 3 that we then need $\delta>4$ to obtain such a high $\Phi_{1.63} / \Phi_{6.13}$ but, again, allowing even moderate source temperatures results in a reduction of fast ion numbers: just $T=2 \times 10^{7} \mathrm{~K}$ lets us reduce $\delta$ to $\simeq 4.5$, and $N_{\mathrm{p}}$ to $\simeq 4.5 \times 10^{36}$, for example. In this case ion numbers and energies are comparable to those deduced assuming a neutral target (more precisely, smaller by a factor of about two). Although the overall importance of fast ions in the flare process is little changed, we still reach significantly different conclusions about the products of the ion accelerator.

All of the above examples rest on our assumed value for the source ${ }^{20} \mathrm{Ne} /{ }^{16} \mathrm{O}$ abundance ratio, a quantity that can evidently vary significantly, even within a single active region (e.g. Schmelz et al. 1996). Other possibilities are opened up if we felt it necessary to insist on a different, particular value for this abundance ratio. And in a real event, other quantities like the observed $\alpha-\alpha$ line fluences would enter the discussion in a more crucial way.
Table 1. Illustrative sets of parameters consistent with $\Phi_{1.63}=18 \mathrm{~cm}^{-2}$ and $\Phi_{6.13} / \Phi_{1.63}=1.4$.

\begin{tabular}{cccccc}
\hline \hline$N_{\alpha} / N_{\mathrm{p}}$ & $\delta$ & $T(\mathrm{~K})$ & $N_{\mathrm{p}}$ & $\begin{array}{c}\text { proton } \\
\text { energy (ergs) }\end{array}$ & $\begin{array}{c}\mathrm{p}+\alpha \\
\text { energy (ergs) }\end{array}$ \\
\hline 0.1 & 4.5 & 0 & $2.7 \times 10^{37}$ & $5.5 \times 10^{31}$ & $7.7 \times 10^{31}$ \\
0.5 & 5.4 & 0 & $3.4 \times 10^{37}$ & $6.5 \times 10^{31}$ & $1.9 \times 10^{32}$ \\
0.1 & 4.0 & $2 \times 10^{7}$ & $6.0 \times 10^{36}$ & $1.5 \times 10^{31}$ & $2.1 \times 10^{31}$ \\
0.1 & 3.5 & $6 \times 10^{7}$ & $1 \times 10^{36}$ & $2.7 \times 10^{30}$ & $3.8 \times 10^{30}$ \\
0.5 & 4.5 & $2 \times 10^{7}$ & $4.5 \times 10^{36}$ & $1 \times 10^{31}$ & $3.1 \times 10^{31}$ \\
\hline
\end{tabular}

Table 1 summarises the properties of the above examples. Energies quoted there are those in ions above $1 \mathrm{MeV} /$ nucleon kinetic energy.

\section{4. ${ }^{20} \mathrm{Ne}$ abundance and partial trapping}

Ramaty et al. (1996) deduced $\delta$ in several flares from the ratio $\Phi_{2.223} / \Phi_{4.44}$. They found consistency with the values of $\delta$ deduced from $\Phi_{1.63} / \Phi_{6.13}$ only by assuming an enhanced ${ }^{20} \mathrm{Ne}$ abundance, ${ }^{20} \mathrm{Ne} /{ }^{16} \mathrm{O}=0.25$ as opposed to the more standard value of 0.14 . Might a warm target enhancement of $\Phi_{1.63}$ reconcile its observed strength with the standard ${ }^{20} \mathrm{Ne}$ abundance, even if containment is not perfect as assumed in the foregoing? 


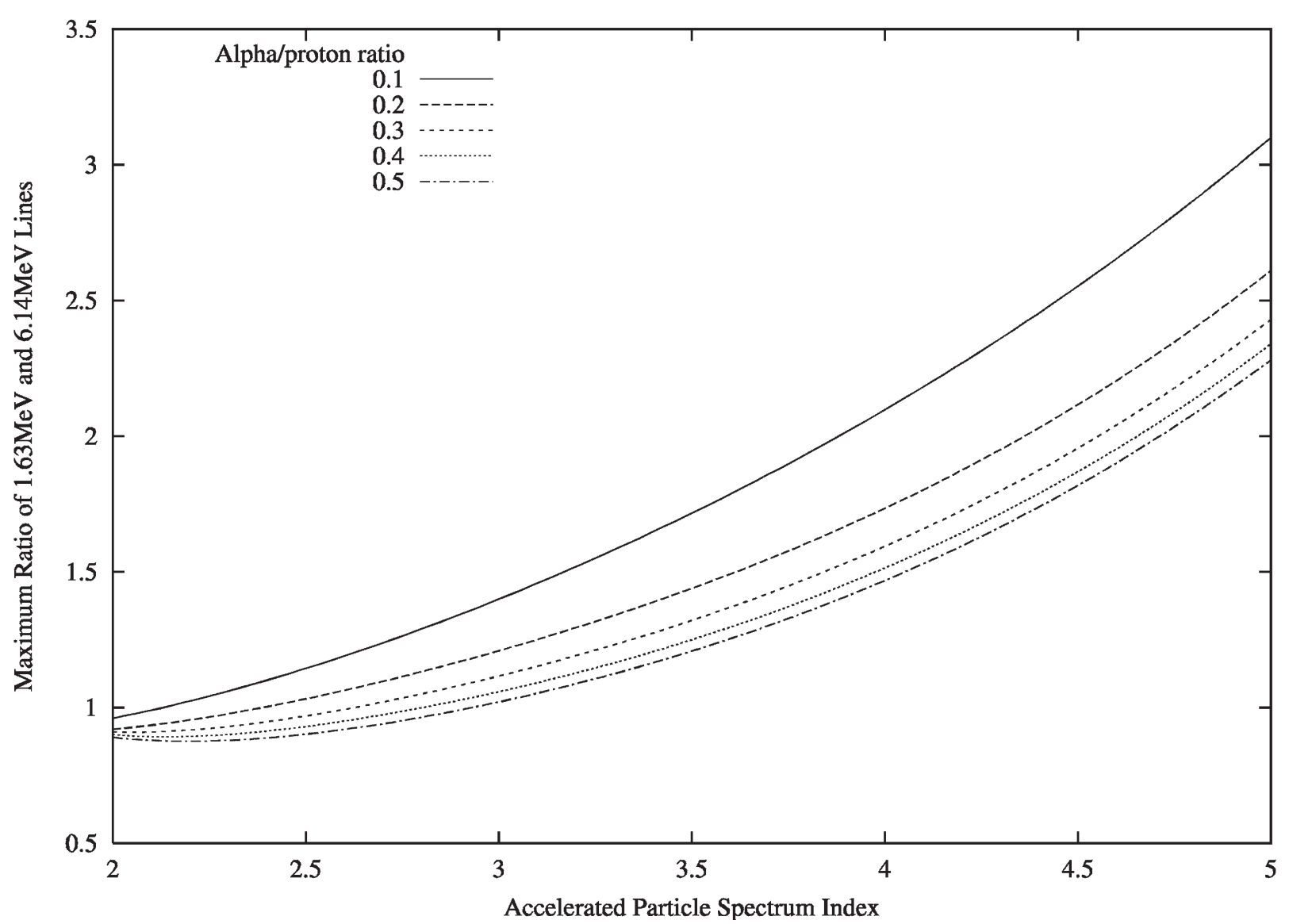

Fig. 3. The maximum value of $\Phi_{1.63} / \Phi_{6.13}$ enhanced by the warm target model as it depends on the accelerated particle spectrum index delta, for a range of values of fast $\alpha$-particle abundance relative to protons.

Figures 1, 2 and 3 together show that the necessary enhancement is certainly possible in principle for a fully contained source. We have also investigated a partial trapping situation in which some ions (possibly those accelerated with pitch angles inside the loss cone) precipitate immediately, while the remainder produce a trapped, warm target yield over a longer period. In such a situation there would be an impulsive component to $\gamma$-ray line emission, as observed, but event-integrated fluences would also include a more gradual, warm target component. The total thick-target yield in any line is then just a linear combination of the warm, ionised and cold, neutral target yields. Suppose for illustration that 0.5 of all accelerated ions are trapped in a coronal warm target, while the remainder precipitate to the cold, neutral atmosphere. Combining the calculations of the warm and cold target yields, we find that $\Phi_{1.63} / \Phi_{6.13}$ can still be enhanced significantly, for instance by $43 \%$ for $\delta=3.75$ and $T=6 \times 10^{7} \mathrm{~K}$. If we interpreted this fluence increase solely as resulting from a ${ }^{20} \mathrm{Ne}$ abundance enhancement, we would deduce ${ }^{20} \mathrm{Ne} /{ }^{16} \mathrm{O}=0.20$ as opposed to the value of 0.14 used in these calculations. Larger fractions of trapped ions would lead to larger apparent ${ }^{20} \mathrm{Ne}$ abundance enhancements.

Suppose now that all ions are contained in a warm target for a period of time significantly shorter than their warm target stopping times but longer than their loop transit times (so that we do not simply revert to the results of Emslie et al. 1997).
Ions will produce only some fraction of their warm, thick target line yields before precipitating. Might the precipitated distribution nevertheless be softened such as to produce enhanced $1.63 \mathrm{MeV}$ flux from the cold neutral target?

A detailed discussion of this question will be presented elsewhere along with a study of warm target line flux temporal evolution. Here we give an approximate treatment containing the essential features.

For few $\mathrm{MeV}$ protons in $10^{7}$ to $10^{8} \mathrm{~K}$ plasmas, the "warm target" energy loss rate approximation of Tamres et al. (1986) applies (see also MacKinnon 1989), and we have approximately

$\frac{\mathrm{d} E}{\mathrm{~d} t}=-0.1 n T^{-3 / 2} E$

Here we have set $\Lambda=25$ as a representative value for the few $\mathrm{MeV}$ energy range (Appendix). This expression does not hold exactly for all ion energies of possible interest, but serves our semi-quantitative purposes here.

Consider now the evolution of the proton distribution in a homogeneous, confined warm target region. Let $N(E, t)$ denote the number of protons in the region per unit proton energy $E$ at time $t$. Solving the continuity equation

$\frac{\partial N}{\partial t}+\frac{\partial}{\partial E}\left[\frac{\mathrm{d} E}{\mathrm{~d} t} N\right]=0$ 
with the energy loss rate of Eq. (5) and initial condition

$N(E, t=0)=A E^{-\delta}$

we find

$N(E, t)=A E^{-\delta} \mathrm{e}^{(1-\delta) K^{\prime} t}$.

Here $K^{\prime}=0.1 n T^{-3 / 2}$. Equation (8) has one slightly surprising feature: protons in the appropriate energy range trapped in a warm target region retain the same energy distribution. Trapping them in a warm target region for some period of time before precipitation will make no important difference to the form of the precipitated energy distribution, only to the numbers of protons found above any given energy. While this evolved distribution is softer than that obtained by letting protons evolve in a cold target, it is not different from the injected distribution.

Suppose that all ions are injected into a fully contained warm target, but that containment breaks down after some time $t$. Because the form of the ion distribution does not change, we may then apply the same linear combination of warm and cold target yields described above to calculate the resulting line fluence ratios. Specifically, after a time $t$, a fraction $1-\mathrm{e}^{(1-\delta) K^{\prime} t}$ of the warm target yield has been produced, and a fraction $\mathrm{e}^{(1-\delta) K^{\prime} t}$ of the neutral target yield from the same energy distribution will subsequently be produced. The results quoted above, for the case that half the ions are trapped in a warm target region and the other half precipitate immediately to the neutral atmosphere, will apply if trapping ceases at a time $\ln 2 /(1-\delta) K^{\prime}$. For example, such a situation applies after $130 \mathrm{~s}$, scaling in inverse proportion to density, with $\delta=3.5$, $n=10^{10} \mathrm{~cm}^{-3}, T=6 \times 10^{7} \mathrm{~K}$. Particularly with coronal densities rather higher than this, breakdown of coronal containment after just a few 10s of seconds will result in an admixture of cold and warm target line yields apparently implying a significantly higher ${ }^{20} \mathrm{Ne}$ abundance. If we are prepared to accept coronal densities in the region of $10^{11} \mathrm{~cm}^{-3}$, this effect may thus offer an explanation of the apparent high ${ }^{20} \mathrm{Ne}$ abundance found in Ramaty et al. (1996), while remaining consistent with impulsive phase time profiles.

\section{Conclusions and discussion}

We have reconsidered the possible role of warm target effects in the interpretation of flare de-excitation $\gamma$-ray line fluxes. Our key finding is that temperatures of just a few $\times 10^{7} \mathrm{~K}$, no higher than flare coronal temperatures diagnosed by other means, are high enough to preferentially increase the coronal lifetimes of significant numbers of the protons which can excite the $1.63 \mathrm{MeV}$ line of ${ }^{20} \mathrm{Ne}$. This effect is lessened when fast alphas are overabundant but still occurs to a significant degree. Effective coronal trapping of flare protons is a necessary precondition for warm target effects to be significant, however; otherwise we revert to the situation treated in detail by Emslie et al. (1997), in which warm target effects were shown to be unimportant. When other factors indicate that such trapping may be occurring, deductions of proton (ion) energy distribution, and/or ${ }^{20} \mathrm{Ne}$ abundance, need to respect this possibility.
It should also be considered in any comprehensive attempt to determine the partitioning of flare energy between mass motion, bulk heating and the acceleration of ions and/or electrons. Trapping of ions in a warm target region for long enough for them to produce at least half of their total, warm target line yield may remove the need for an anomalously high ${ }^{20} \mathrm{Ne}$ abundance.

We recalled various factors that might contain a significant fraction of flare-accelerated protons in the corona. Most important is the possibility that the proton distribution itself drives unstably growing Alfvén waves. Protons will inevitably become anisotropic as they travel away from a localised flaring region, and only a modest anisotropy is needed for unstable wave growth with typical $\gamma$-ray producing proton numbers (Tamres et al. 1989). This self-containing behaviour seems difficult to avoid in large flares. At least in one SMM flare its occurrence seems almost inescapable (Smith \& Brecht 1991), unless MHD turbulence plays no role in ion acceleration.

We also need, however, to acknowledge that various other findings argue against a coronally contained, nearly isotropic ion distribution, at least in some flares. Observations of redshifted lines argue that the fast ion pitch-angle distribution is significantly anisotropic, consistent with precipitation of most ions into the photosphere (Share et al. 2002; Smith et al. 2003). An ion population self-contained via MHD turbulence would still exhibit some residual anisotropy (Kulsrud \& Pearce 1969) but detailed discussion of the magnitude of this effect lies outside the scope of this paper.

The magnitude of fast ion stopping times, easily in excess of $100 \mathrm{~s}$ for $\gamma$-ray producing ions in coronal conditions, also constitute a potential difficulty for any model which involves coronal production of $\gamma$-rays. De-excitation $\gamma$-ray lines exhibit at least a component that is clearly impulsive in nature (e.g. Chupp 1984), suggesting ions stop on shorter timescales, and detailed modelling apparently requires rapid ion precipitation (Hua et al. 1989). For the modest temperatures suggested in Sect. 3.3, warm target effects are not significantly worse off in this respect. The relative contributions to total flare $\gamma$-ray line fluences of impulsive and gradual components remain uncertain, apparently, so any further discussion is probably premature at this point. We note that sources with partial containment or containment that ceases to be effective at some point might allow warm target effects to be important for interpreting line fluences without contradicting observed temporal behaviour. The warm target source studied here will display a characteristic sort of behaviour, with lines sensitive to lower energy ions decaying more gradually than in a cold target source. Detailed modelling of the expected temporal behaviour may yield tests of these ideas, and will be carried out elsewhere.

So far all detected solar $\gamma$-rays have been from large flares. No doubt we are seeing only the large event end of the distribution of $\gamma$-ray flare sizes. Conclusions about the likelihood of proton self-containment also rest, thus far, on analyses of large flares. In more modest events fast protons may be sufficiently dilute not to drive unstable Alfvén wave growth, and proton containment in hot regions may only occur to a lesser degree. A dependence on flare size of $\Phi_{1.63} / \Phi_{6.13}$ would argue strongly in favour of the scenario outlined here. 
Acknowledgements. Mark Toner's work has been partially supported by PPARC. We are grateful to John Brown and Lyndsay Fletcher for useful questions and comments, to Ron Murphy for very helpful correspondence and to Giulio del Zanna for a useful conversation on the $\mathrm{Ne}$ abundance. The anonymous referee's constructive criticism significantly improved the paper, in particular encouraging us to consider partially trapped situations. All work on these topics is hugely indebted to the efforts of the late Reuven Ramaty and his various collaborators, including Natalie Mandzhavidze.

\section{Appendix A: Effective Coulomb logarithm in neutral and ionised targets}

The rate of loss of energy, and thus the penetrating power, of fast ions is a well-studied topic. A recent review (Weaver \& Westphal 2002) summarises results in the energy range appropriate here as well as concentrating on effects that become important for heavy ions at relativistic energies.

Equations (2) and (3) summarise the rate at which the energy $E$ of a fast proton changes in an ionised medium. An expression of this form also applies in the case of energy loss in a neutral medium, via the introduction of an "effective Coulomb logarithm", given explicitly by e.g. Mott \& Massey (1949), Emslie (1978). For protons in the nonrelativistic regime, its value is given numerically by

$\Lambda_{\text {neutral }}=5+\ln E$.

Here $E$ is measured in $\mathrm{MeV}$. It is easy to verify that the energy loss rate given by Eqs. (2), (3) and (A.1) is in excellent agreement with the empirical findings of Barkas \& Berger (1964), at least for the energy range needed here (see also Weaver $\&$ Westphal 2002). Comparison of the radiative properties of ionised and neutral targets may thus be carried out via comparison of the effective Coulomb logarithms.

Discussions of fast electron stopping carried out to understand deka-keV X-rays (Brown 1971) generally set the Coulomb logarithm equal to a constant. This habit would be a bad one here: $\Lambda$ varies from 5 to almost 10 in the relevant 1-100 MeV energy range, and its energy-dependence is key to theoretical understanding of the empirical (Barkas \& Berger 1964) results.

For protons in an ionised medium, in the absence of too strong a magnetic field (Emslie 1978),

$\Lambda_{\text {ionised }}=\ln \frac{m_{\mathrm{e}} v^{3}}{e^{2} v_{\mathrm{p}}}$.

Here $v_{\mathrm{p}}$ is the plasma frequency. Numerically,

$\Lambda_{\text {ionised }}=23.2+\frac{3}{2} \ln E+\frac{1}{2} \ln n_{10}$

where $n_{10}$ measures ambient density in units of $10^{10} \mathrm{~cm}^{-3}$. In a medium of $10^{10} \mathrm{~cm}^{-3}$ density, $\Lambda_{\text {ionised }}$ varies from 23.2 to 30.1 ; it takes values in excess of four times larger than $\Lambda_{\text {neutral }}$, but varies less over the relevant energy range.

\section{References}

Barat, C., Trottet, G., Vilmer, N., et al. 1994, ApJ, 425, L109
Barkas, W. H., \& Berger, M. J. 1964, Tables of Energy Losses and Ranges of Heavy Charged Particles, NASA SP-3013

Bespalov, P. A., Zaitsev, V. V., \& Stepanov, A. V., 1987, Sol. Phys., 114,127

Brown, J. C. 1971, Sol. Phys., 18, 489

Cameron, A. G. W. 1973, Space Sci. Rev., 15, 121

Chupp, E. L. 1984, ARA\&A, 22, 359

Dyer, P., Bodansky, D., Seamster, A. G., Norman, E. B., \& Maxson, D. R. 1981, Phys. Rev. C, 32, 5, 1865

Dyer, P., Bodansky, D., Leach, D. D., Norman, E. B., \& Seamster, A. G. 1985, Phys. Rev. C, 32, 6, 1873

Emslie, A. G. 1978, ApJ, 224, 241

Emslie, A. G., Brown, J. C., \& MacKinnon, A. L. 1997, ApJ, 485, 430

Fletcher, L., \& Martens, P. C. H. 1998, ApJ, 505, 418

Hoyng, P., Brown, J. C., \& van Beek, H. F. 1976, Sol. Phys., 48, 197

Hua, X.-M., Ramaty, R., \& Lingenfelter, R. 1989, ApJ, 516, 532

Hulot, E., Vilmer, N., Chupp, E. L., Dennis, B. R., \& Kane, S. R. 1992, A\&A, 256, 273

Kennel, C. F., \& Petschek, H. E. 1966, JGR, 71, 1

Kocharov, L., Kovaltsov, G. A., \& Torsti, J. 1999, ApJ, 519, 422

Kocharov, L., Kovaltsov, G. A., \& Torsti, J. 2000, ApJ, 543, 438

Kozlovsky, B., Murphy, R. J., \& Ramaty, R. 2002, ApJS, 141, 523

Kulsrud, R., \& Pearce, W. M. 1969, ApJ, 156, 445

Lau, Y.-T., Northrop, T. G., \& Finn, J. M. 1993, ApJ, 414, 908

Lin, R. P., \& Hudson, H. S. 1976, Sol. Phys., 50, 153

MacKinnon, A. L. 1989, A\&A, 226, 284

Miller, J. A., \& Ramaty, R. 1987, Sol. Phys., 113, 195

Mott, N. F., \& Massey, H. S. W. 1949, Theory of Atomic Collisions, 2nd. ed. (Oxford)

Murphy, R. J., \& Ramaty, R. 1984, Adv. Space Res., 4, 7, 127

Murphy, R. J., Hua, X.-M., Kozlovsky, B., \& Ramaty, R. 1990, ApJ, 351,229

Murphy, R. J., Ramaty, R., Koslovsky, B., \& Reames, D. V. 1991, ApJ, 371,793

Murphy, R. J., Share, G. H., Grove, J. E., et al. 1997, ApJ, 490, 883

Ramaty, R., Kozlovsky, B., \& Lingenfelter, R. E. 1979, ApJS, 40, 487

Ramaty, R. 1986, in Physics of the Sun, vol. 2, ed. P. A. Sturrock, et al. (Dordrecht: Reidel)

Ramaty, R., Mandzhavidze, N., \& Kozlovsky, B. 1996, in High Energy Solar Physics, ed. R. Ramaty, N. Mandzhavidze, \& X.-M. Hua (AIP), 172

Seamster, A. G., Norman, E. B., Leach, D. D., Dyer, P., \& Bodansky, D. 1984, Phys. Rev. C, 29, 2, 394

Schmelz, J. T., Saba, J. L. R., Ghosh, D., \& Strong, K. T. 1996, ApJ, 473,519

Share, G. H., \& Murphy, R. J. 1995, ApJ, 452, 933

Share, G. H., \& Murphy, R. J. 1998, ApJ, 508, 876

Share, G. H., Murphy, R. J., Kiener, J., \& de Séréville, N. 2002, ApJ, 573,464

Smith, D. F. 1990, Basic Plasma Processes on the Sun, IAU Symp., 142,375

Smith, D. F., \& Brecht, S. H. 1991, ApJ, 373, 289

Smith, D. M., Share, G. H., Murphy, R. J., et al. 2003, ApJL, submitted

Spicer, D. S., \& Emslie, A. G. 1988, ApJ, 330, 997

Spitzer, L. 1956, Physics of Fully Ionized Gases (New York: Interscience)

Tamres, D. H., Canfield, R. C., \& McClymont, A. N. 1986, ApJ, 309, 409

Tamres, D. H., Melrose, D. B., \& Canfield, R. C. 1989, ApJ, 342, 576

Trubnikov, B. A. 1965, Rev. Plasma Phys., 1, 205

Tsuneta, S., Masuda, S., Kosugi, T., \& Sato, J. 1997, ApJ, 478, 787

Vestrand, W. T., \& Forrest, D. J. 1993, ApJ, 409, L69

Warren, H. P., \& Reeves, K. K. 2001, ApJ, 554, L103

Weaver, B. A., \& Westphal, A. J. 2002, Nucl. Instr. Meth. B, 187, 285 